\title{
Von Vulkan zu Vulkan - Inselhopping auf den Liparischen Inseln
}

\author{
Hilke Steinecke \& Peter Schubert
}

\begin{abstract}
The Aeolian Islands (or Lipari Islands) are located to the northeast of Sicily and are of volcanic origin. Stromboli, with its permanently active volcano, is most widely known. Each of the seven islands is unique in its own right. The flora and geology of the individual islands are briefly introduced here. Having been listed as a UNESCO World Heritage Site in the year 2000, the islands have fortunately not been negatively affected by mass tourism so far.
\end{abstract}

\section{Zusammenfassung}

Die Liparischen Inseln sind der Nordküste Siziliens vorgelagert und vulkanischen Ursprungs. Die bekannteste Insel ist Stromboli mit gleichnamigem Vulkan, der permanent aktiv ist. Alle sieben Inseln haben ihr eigenes Gesicht. Die einzelnen Inseln werden hinsichtlich ihrer Pflanzenwelt und Geologie vorgestellt. Sie wurden im Jahr 2000 in die Liste der UNESCO-Weltkulturerbe aufgenommen und sind bisher glücklicherweise von Negativeigenschaften eines Massentourismus verschont geblieben.
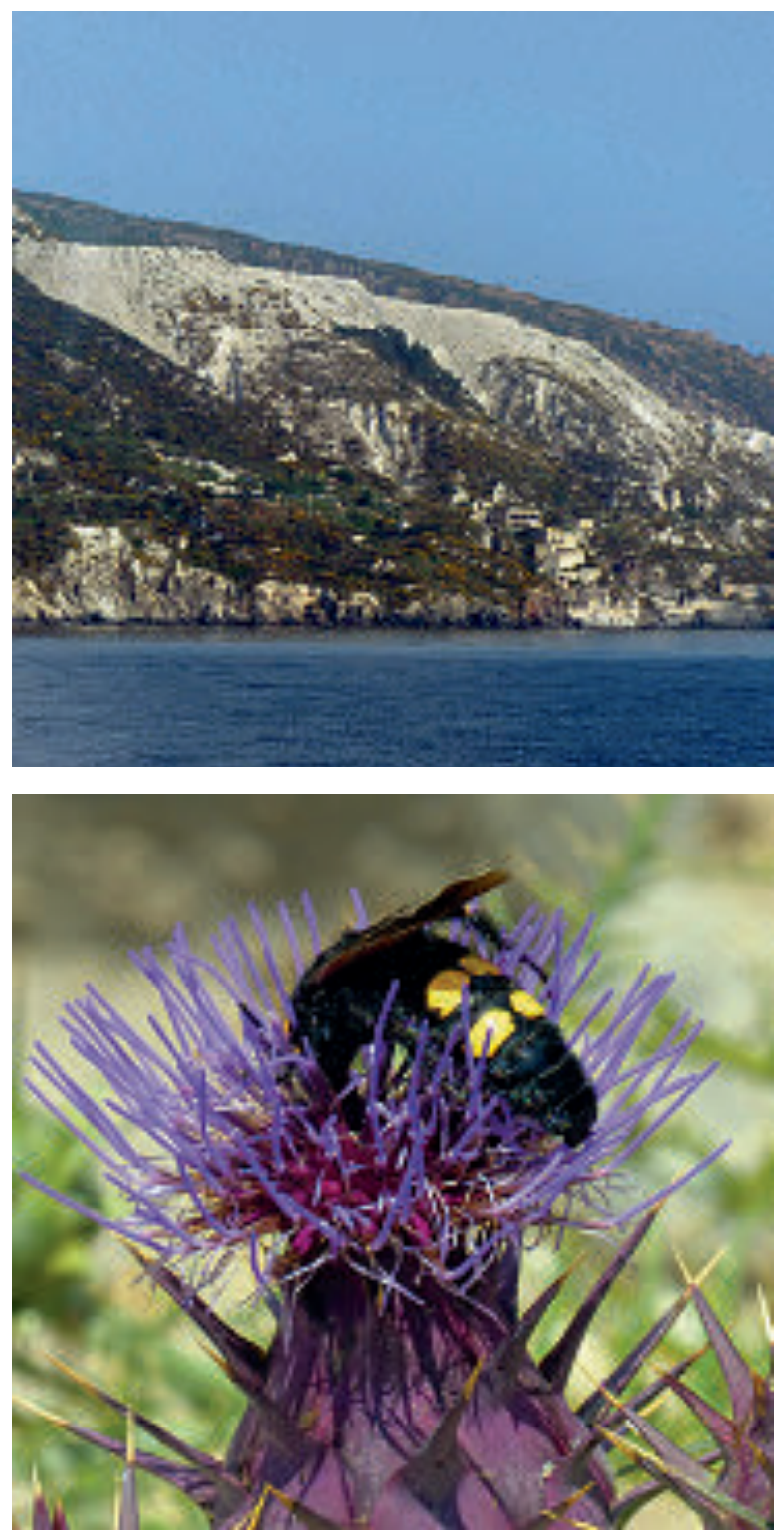

\section{Einführung}

Im Norden Siziliens liegen in einer Entfernung von $30-80 \mathrm{~km}$ vor der Küste im Tyrrhenischen Meer die Liparischen Inseln. Sie werden auch Äolische Inseln genannt und heißen auf Italienisch Isole Eolie. Laut griechischer Mythologie wohnt Äolus, der Gott der Winde, auf einer dieser Inseln. Die Inseln sind durch vulkanische Aktivität entstanden. In ihrem Bereich schiebt sich die afrikanische Kontinentalplatte unter die eurasische. Filicudi ist die älteste Insel, gefolgt von Panarea und Salina. Die jüngsten Inseln sind Vulcano und Stromboli. Außer den sieben sichtbaren Inseln existieren zudem fünf zusätzliche unterirdische Vulkane. Die Liparischen Inseln sind für die weltweite Vulkanforschung ein Musterbeispiel und von großer Bedeutung. Die Äolischen Inseln gehören seit 2000 zum UNESCO-Weltnaturerbe und unterliegen deshalb strengen Schutzrichtlinien.

Abb. 1 (oben): Ehemaliger Bimsabbau auf Lipari.

Abb. 2 (unten): Blütenkopf von Onopordum illyricum mit Dolchwespe.

Abb. 3 (Seite 89 oben): Blick über die schroffe Küste von Lipari nach Vulcano.

Abb. 4 (Seite 89 unten): Kaolingrube auf Lipari, im Vordergrund Blüten von Onopordum illyricum. Die Blätter von Artemisia arborescens schimmern silbergrau. 


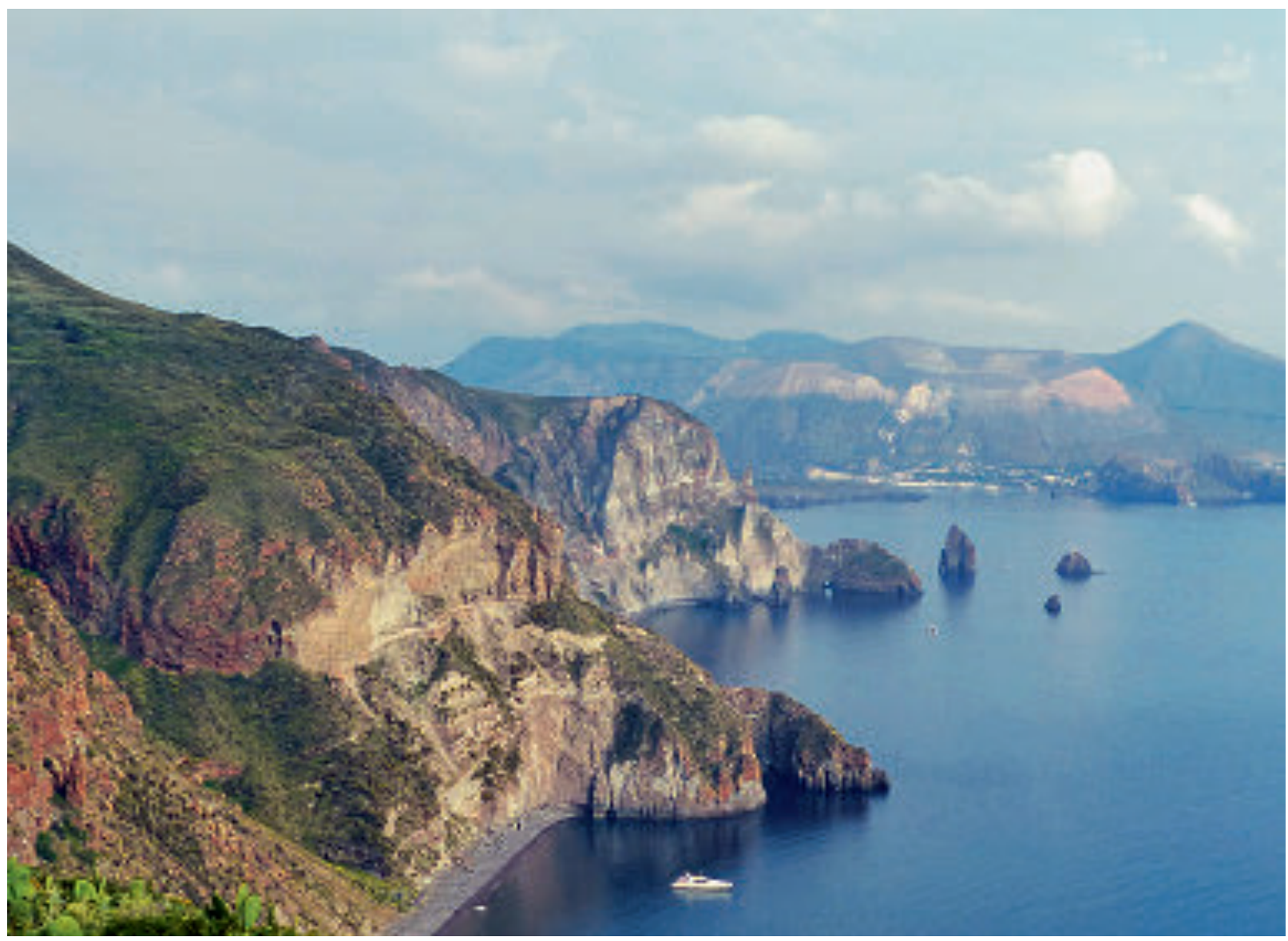

Die Hauptinsel der sieben Eilande ist die namensgebende Insel Lipari. Berühmt wegen ihres formschönen Vulkans mit kreisrundem Krater ist Vulcano. Auf den Namen dieser Insel ist die allgemeine Bezeichnung Vulkan für einen feuerspeienden Berg zurückzuführen. Die vermutlich beeindruckendste und weltweit bekannteste Insel des Archipels ist Stromboli mit ihrem permanent feuerspuckenden Vulkan. Panarea ist die kleinste Insel, landschaftlich sehr reizvoll und von wohlhabenden Italienern als Urlaubsdomizil auserwählt. Salina wird durch zwei markante, in etwa gleichgroße Vulkankegel dominiert. Filicudi und Alicudi liegen etwas abseits westlich der übrigen Inseln.

Erreichbar sind die Inseln mit Fähren von verschiedenen Häfen in Italien, so von Neapel, Messina oder Tropea in Kalabrien. Die meisten Touristen reisen (über Catania) von Milazzo im Norden Siziliens auf die Liparischen Inseln. Von hier aus gibt es auch die meisten und schnellsten Fährverbindungen (Autofähre, Tragflächenboote), die mehrmals täglich die

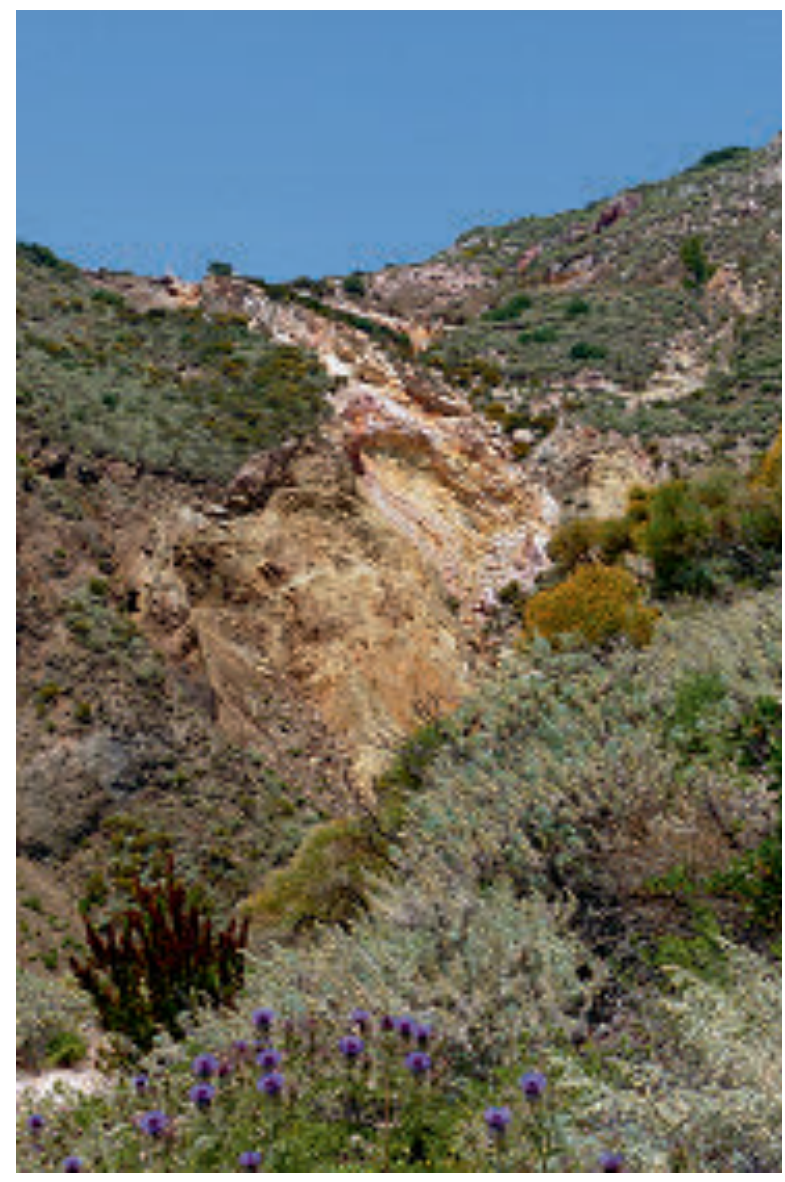




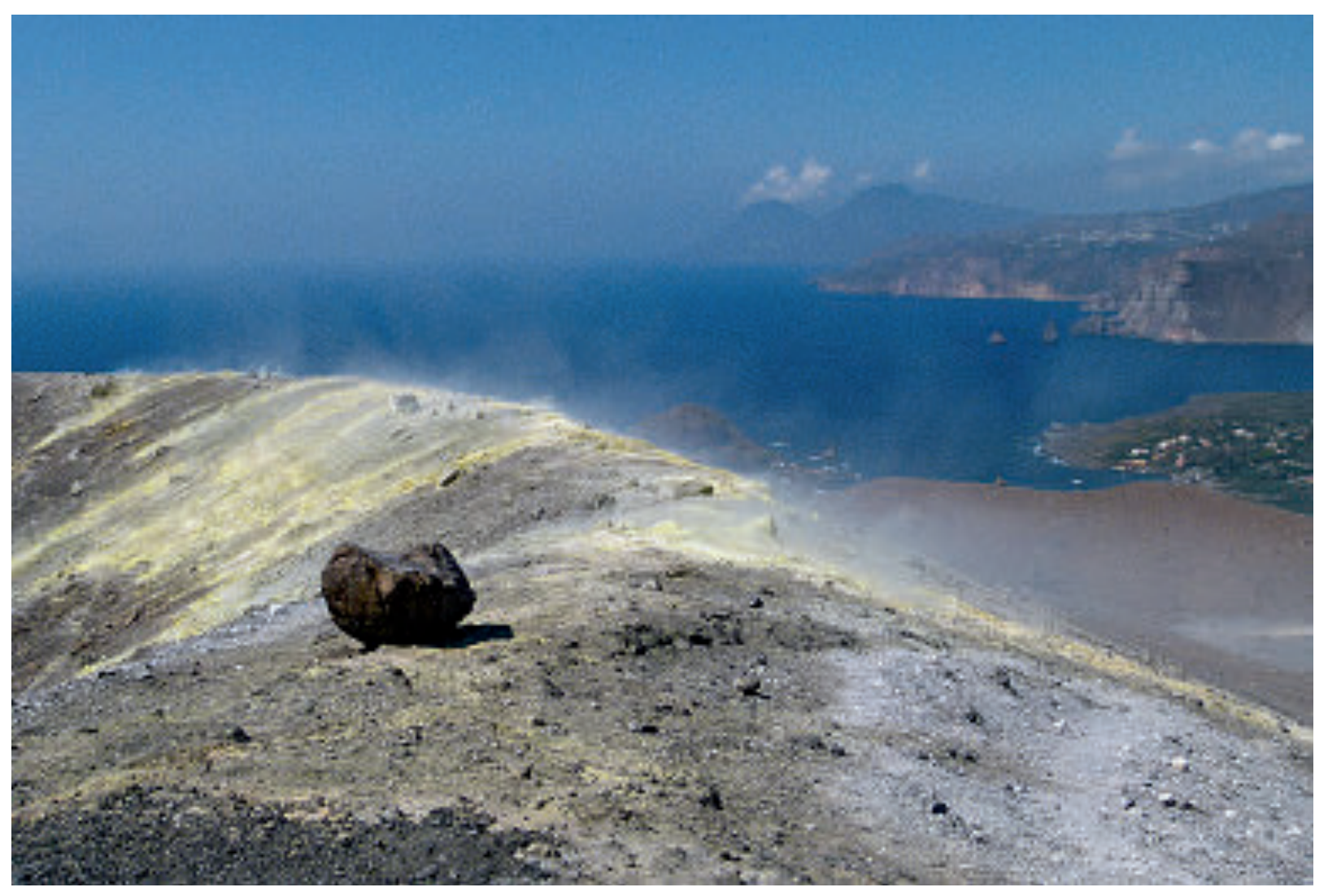

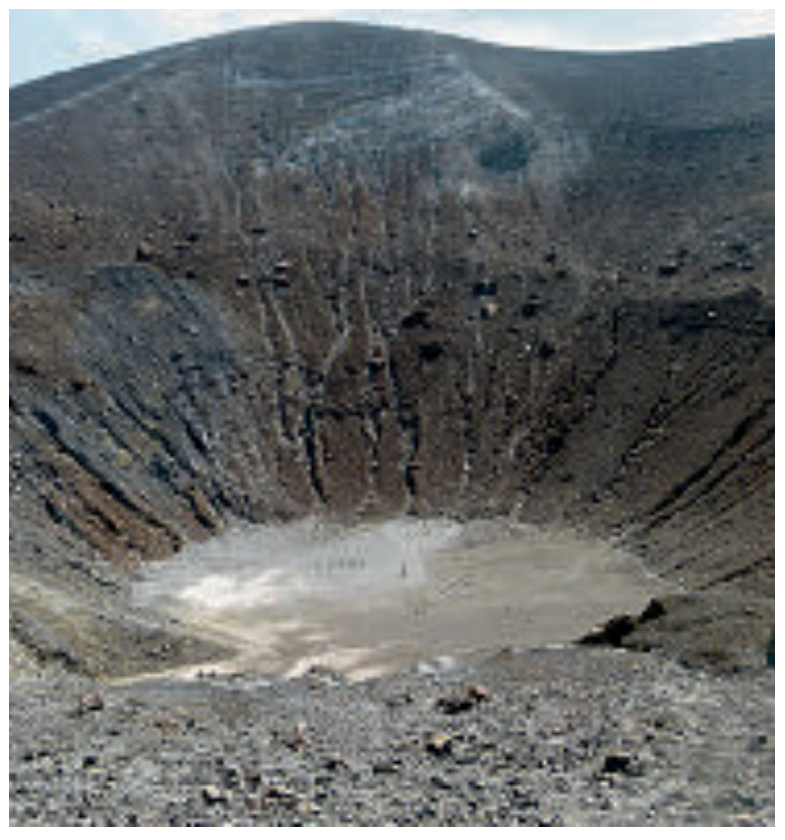

Abb. 5 (oben): Blick vom schwefeligen Kraterrand auf Vulcano nach Lipari und Salina.

Abb. 6 (unten): Blick in den großen Krater von Vulcano.

Abb. 7 (Seite 91 oben): Pancratium maritimum auf Vulcano.

Abb. 8 (Seite 91 unten): Salina, von Lipari aus gesehen. verschiedenen Inseln ansteuern. Im Allgemeinen sucht man während einer Reise auf die Isole Eolie mehrere Inseln auf. Vor Ort ist ein Auto nicht nötig, da die Entfernungen zu bestimmten Zielen relativ gering sind, auf den größeren Inseln gute Busverbindungen bestehen oder sowieso überhaupt gar keine Autos vorhanden sind. Jede Insel hat ihren ganz eigenen Charakter, der vor allem durch den Vulkanismus geprägt ist. Hinzu kommt eine bunte Pflanzenwelt aus Wild- und Kulturpflanzen, die sich natürlich abhängig von der Jahreszeit ganz unterschiedlich präsentiert. Nachfolgend sollen die einzelnen Liparischen Inseln - außer Alicudi - unter besonderer Berücksichtigung ihrer Pflanzenwelt und Geologie kurz vorgestellt werden.

\section{Lipari}

Die Hauptstadt der Insel sowie des gesamten Archipels (nur Salina ist eigenständig) ist Lipari. Die Insel Lipari ist - wie auch die übrigen Inseln - vulkanischen Ursprungs, wobei die Entstehung in verschiedenen Phasen erfolgte. Markant sind ihre beiden Vulkanberge 
Monte Chirica und Monte S. Angelo. Im Nordosten gibt es massive Ablagerungen aus Bims, die bereits von der Fähre aus an ihrer hellgrauen Farbe gut zu erkennen sind. Der Bims wurde hier bis 2007 abgebaut. Seitlich der Bimsschichten befinden sich am Monte Pilato Ströme aus sog. vulkanischem Glas (Obsidian), das hier schon in der Jungsteinzeit und später auch von Griechen und Römern abgebaut wurde. Das scharfkantige, glänzend schwarze Material eignet sich gut zur Herstellung von Messern, Jagdgeräten und anderen Werkzeugen. In der Altstadt von Lipari werden in zahlreichen Souvenirgeschäften Obsidianstücke verschiedenster Qualitäten angeboten. Zudem gibt es Werkstätten, in denen aus Obsidian hochwertiger Schmuck hergestellt wird. Häufig treten auf Lipari Rhyolithe auf. Dabei handelt es sich um ein dem Granit entsprechendes vulkanisches Gestein. Seine veraltete Bezeichnung ist Liparit, benannt nach der Insel Lipari. Rhyolithe können in verschiedenen Farben auftreten, bekannt
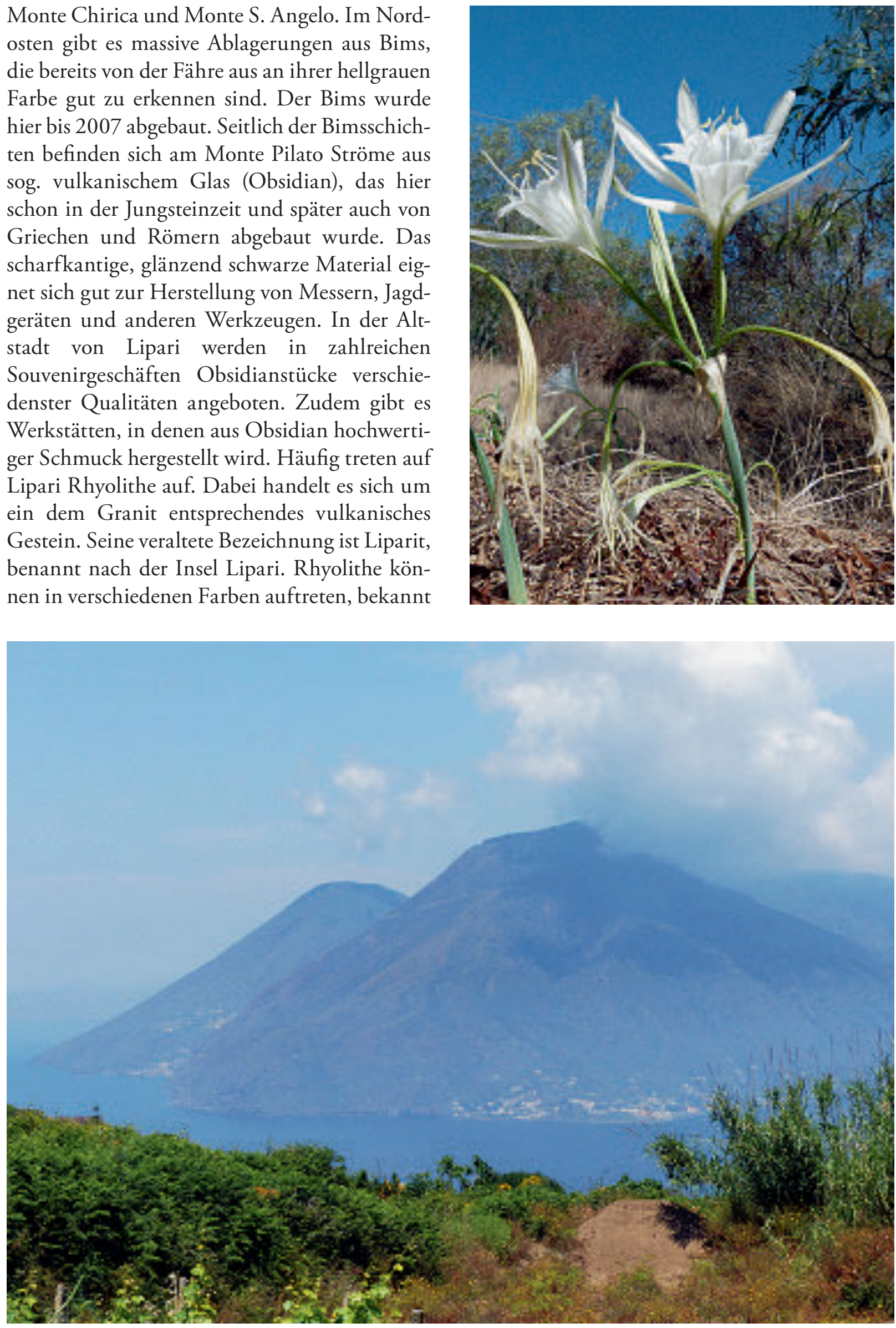


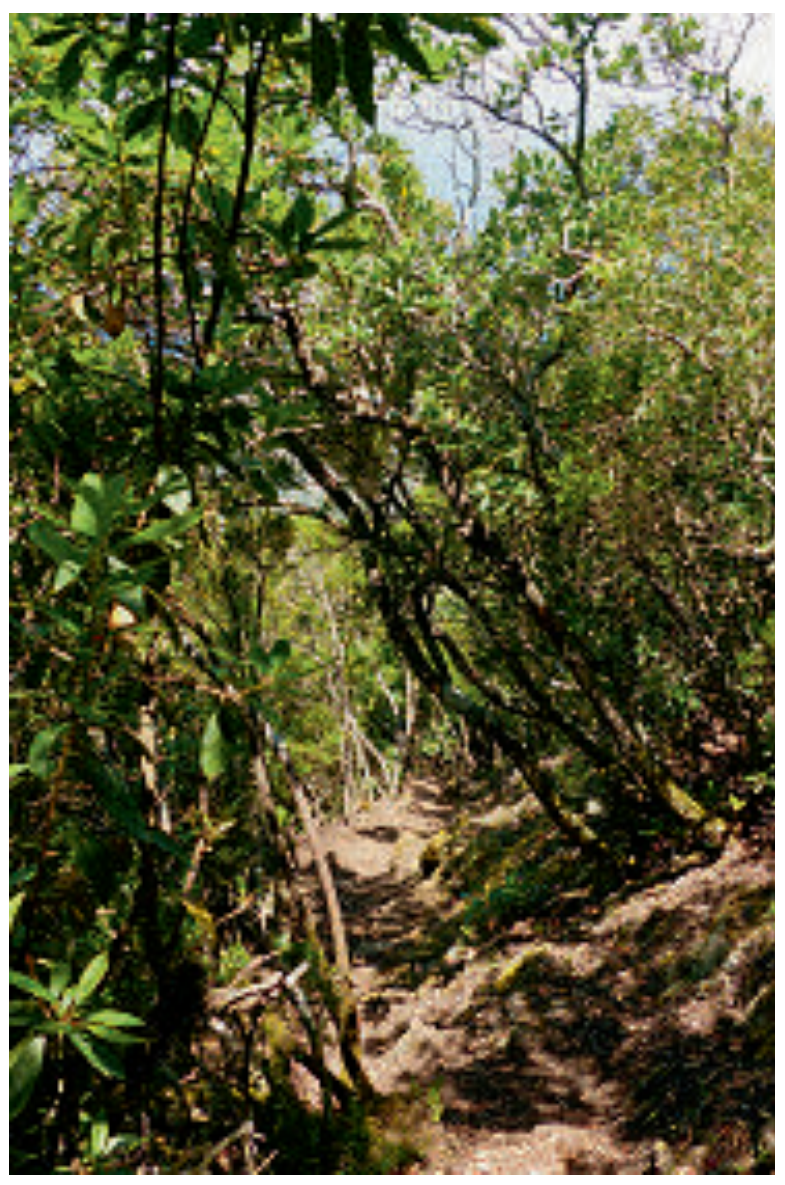

und sehr markant ist die bunte Rhyolith-Vulkanlandschaft in Landmannalaugar auf Island.

Als Hauptinsel und zentraler Ausgangspunkt für Ausflüge auf andere Inseln wird Lipari besonders häufig von Touristen frequentiert. Die hübsche Inselhauptstadt ist mit ihren vielen schmalen alten Gassen sehr einladend. In Gärten und Straßen gedeihen viele Zierpflanzen, die man auch an anderen Orten im Mittelmeergebiet finden kann, so z. B. auch JacarandaBäume. Die Nähe zum Meer mit dem damit verbundenen besonders ausgeglichenen Klima sowie die hohe Strahlungsintensität lassen hier die Blütenfarben besonders intensiv erscheinen. Am Burgberg aus dem 16. Jahrhundert gedeihen riesige, alte Oleander.

Eine geologische Attraktion sind die Kaolingruben, in denen früher Kaolin, das für die Herstellung von Porzellan benötigt wurde, abgebaut wurde. Der canyonartige Geländeeinschnitt schimmert in verschiedenen Farben, zudem gibt es hier noch kleine aktive Fumarolen. Im Sommer kann sich hier die Hitze ziemlich stauen.
Aber trotz Trockenheit und hoher Temperaturen hat sich hier eine üppige Vegetation entwickelt. Besonders markant ist dabei die Illyrische Eselsdistel (Onopordum illyricum), deren violette große Blütenköpfe vielen Insekten Nahrung bieten. Mit ihren langen Pfahlwurzeln erreicht sie auch Wasser, das sich in tieferen Bodenschichten befindet. In Australien wurde sie im 19. Jahrhundert eingeführt und gilt dort heute als invasiver Neophyt sowie lästiges Weideunkraut. Häufig wird die Illyrische Eselsdistel von großen, auffällig schwarz-gelb gefärbten Dolchwespen besucht. Scolia flavifrons beispielsweise hat eine Körperlänge von $4 \mathrm{~cm}$. Dolchwespen ernähren sich von Nektar, und die größeren Arten gehören zu den größten Hautflüglern überhaupt. In Meeresnähe gedeiht auch die Europäische Zwergpalme (Chamaerops humilis), die hier allerdings meist stammlos bleibt, da die Palmen von Ziegen befressen werden.

Im Norden ergeben sich sehr schöne Blicke auf die Nachbarinsel Salina, im Süden kann man von einem Aussichtspunkt an der Straße oberhalb von Lipari eindrucksvolle Blicke auf die nahegelegene Insel Vulcano genießen.

\section{Vulcano}

Vulcano und Stromboli sind die jüngsten und vulkanisch noch aktivsten Inseln des Archipels. Vulcano ist zusammen mit dem Südteil von Lipari entstanden. Im Alten Rom glaubte man, dass Vulcano die Schmiede des Feuergottes Vulcanus sei. Der letzte Ausbruch auf Vulcano ereignete sich im Jahr 1890. Hauptattraktion der Insel ist die knapp $400 \mathrm{~m}$ hohe Fossa, ein Vulkankegel mit zentralem, kreisrundem Krater, der vor rund 10000 Jahren auf einem älteren Vulkan entstanden ist. Der Vulkan ist noch nicht tot, schlummert momentan nur und scheidet im Kraterbereich aus dampfenden, heißen Fumarolen reichlich Schwefel aus, sodass der Kraterrand stellenweise leuchtend schwefelgelb gefärbt ist. Aufgrund der schwefelhaltigen Dämpfe ist der Kraterrand sehr lebensfeindlich, sodass hier keinerlei Pflanzen gedeihen. Der Abstieg in den Krater ist verboten, da sich hier unbemerkt tödlich giftige Gase ansammeln können. Lebensfeindlich geht es auch auf dem 

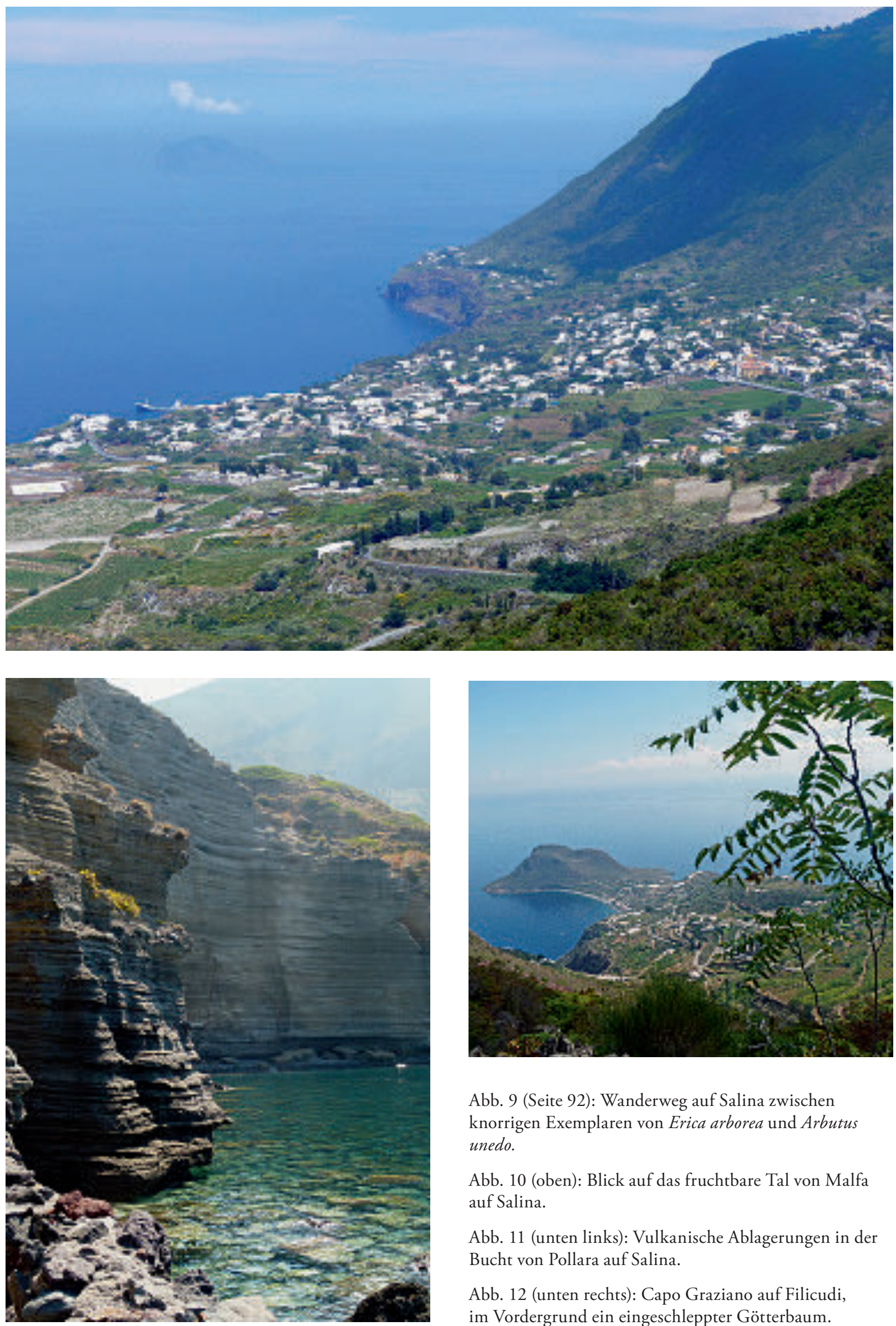

Abb. 9 (Seite 92): Wanderweg auf Salina zwischen knorrigen Exemplaren von Erica arborea und Arbutus unedo.

Abb. 10 (oben): Blick auf das fruchtbare Tal von Malfa auf Salina.

Abb. 11 (unten links): Vulkanische Ablagerungen in der Bucht von Pollara auf Salina.

Abb. 12 (unten rechts): Capo Graziano auf Filicudi, im Vordergrund ein eingeschleppter Götterbaum. 


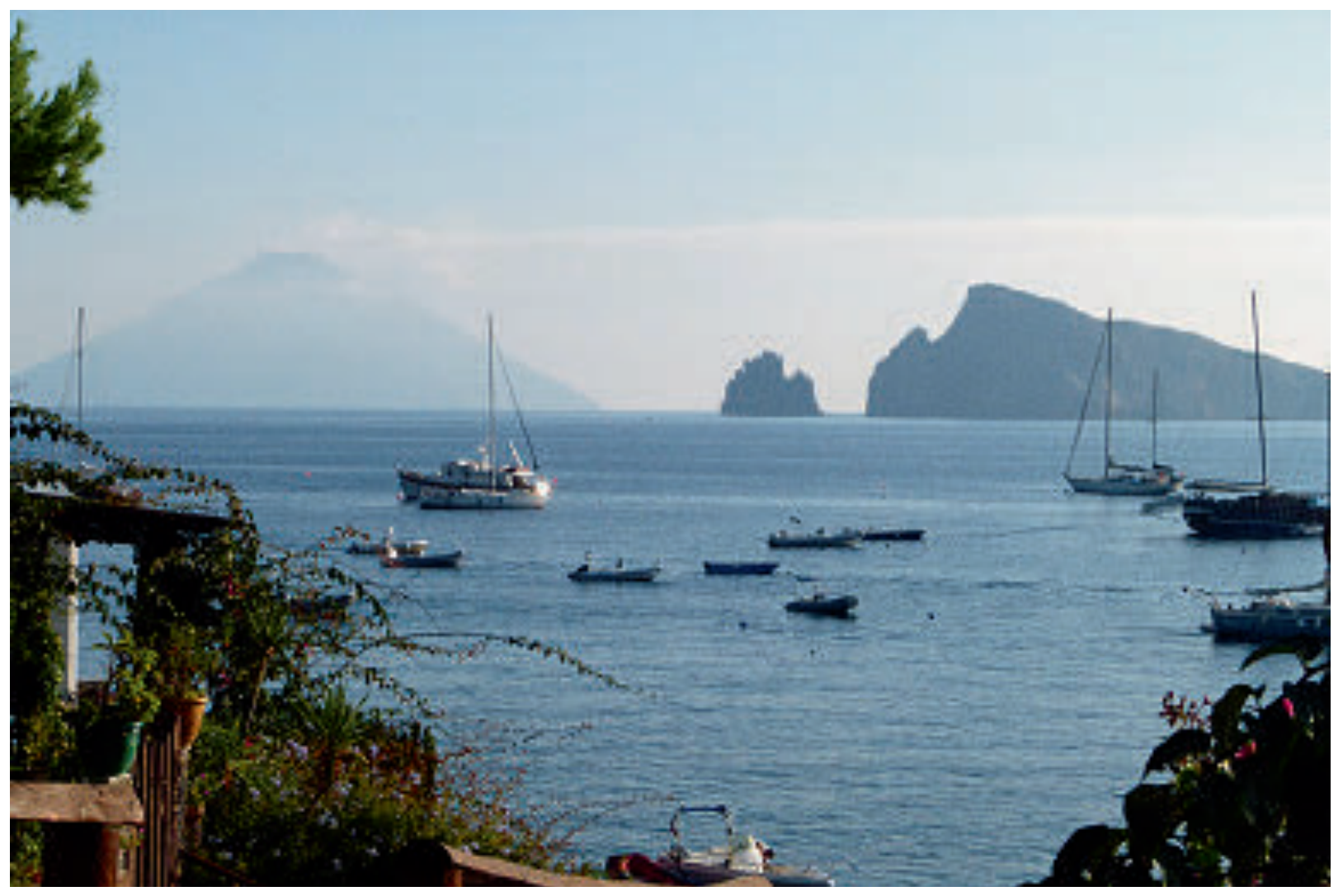

„Toten Feld“ zwischen der Fossa und dem Vulcanello (ein „Inselzipfel“ mit drei kleinen ineinander geschachtelten Vulkankratern) zu. In den Jahren 1913-1916 weitete sich hier die Fumarolentätigkeit aus und es entstanden heiße Schlammlöcher. Alle hier wachsenden Pflanzen fielen den heißen, giftigen Dämpfen zum Opfer. Im Bereich der Schlammquellen wurde eine Badeanstalt eingerichtet, die heutzutage von Touristen reichlich frequentiert wird.

Außerhalb der Bereiche sichtbarer vulkanischer Aktivität ist die Insel vor allem von Strauchvegetation überzogen. Im Frühsommer blühen an den Hängen üppig verschiedene Ginster, darunter auch der Mittelmeer-Ginster (Spartium junceum), sodass die Landschaft leuchtend gelb wirkt. Hier kommt auch der Ätna-Ginster (Genista aetnensis) vor. Dazwischen gedeihen verschiedene Zistrosen wie Cistus incanus und $C$. salvifolius. Im Herbst blühen in Strandnähe auf Sandboden an verschiedenen Stellen die Trichter-Narzissen (Pancratium maritimum). Die großen, weißen Blüten des Zwiebelgewächses haben lange Kronröhren und werden von Nachtfaltern bestäubt.

\section{Salina}

Salina ist die zweitgrößte Insel der Liparischen Inseln, ihr Hauptort ist das beschauliche $\mathrm{Ha}$ fenstädtchen Santa Maria Salina. Salina ist die Insel mit zwei charakteristischen Bergen, weshalb man sie auch sehr leicht aus der Ferne anhand ihrer Silhouette identifizieren kann. Eigentlich handelt es sich sogar um sechs Vulkane, nur sind vier von ihnen deutlich kleiner als die zwei markanten Vulkankegel. Die letzten Vulkanausbrüche fanden hier vor etwa 13000 Jahren statt. Früher wurde aus Salzseen im Südosten der Insel Salz gewonnen, worauf sich der Name Salina bezieht. Heute erinnert andeutungsweise nur noch eine kleine Salzlagune bei Lingua an diesen Wirtschaftszweig.

Salina hat mit ihren beiden Zwillingsvulkanen Monte Fossa delle Felci (962 m) und Monte dei Porri $(860 \mathrm{~m})$ die höchsten Berge des Archipels. Dementsprechend verfangen sich hier am häufigsten die Wolken und regnen sich auch regelmäßig ab. Auf Salina gibt es Süßwasserquellen, weshalb die Insel bereits zur Bronzezeit besiedelt war. Die relativ gute Wasserversorgung fördert eine üppige Vegetation: die reich- 


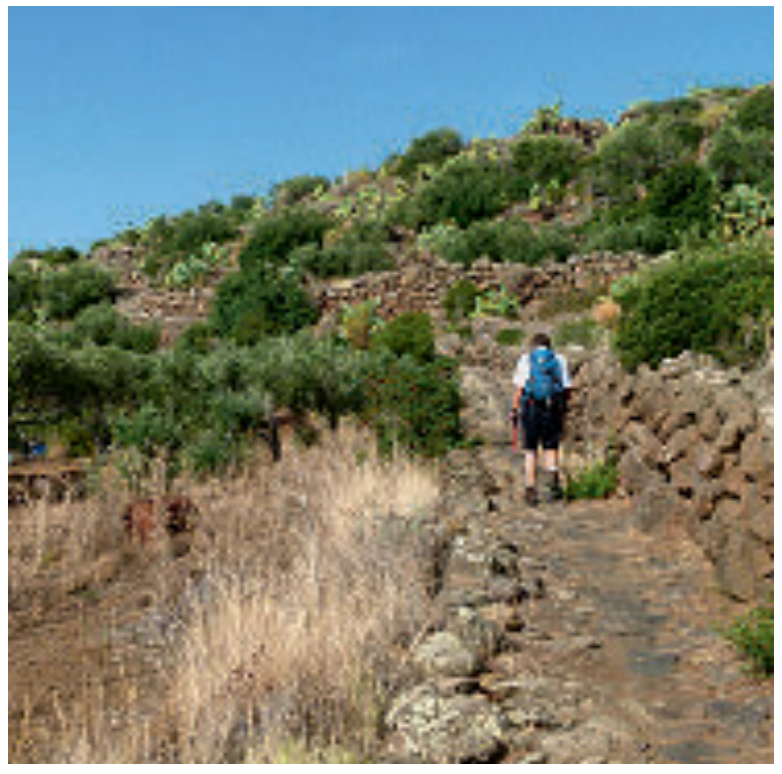

Abb. 13 (Seite 94): Blick von Panarea auf die der Insel vorgelagerten Felsen und Stromboli.

Abb. 14 (links): Pflasterweg durch altes Kulturland mit Ölbäumen und Feigenkakteen auf Filicudi.

Abb. 15 (rechts): Punta Milazzese auf Panarea mit Resten einer bronzezeitlichen Siedlung.

Abb. 16 (unten): Urginea maritima auf Panarea.

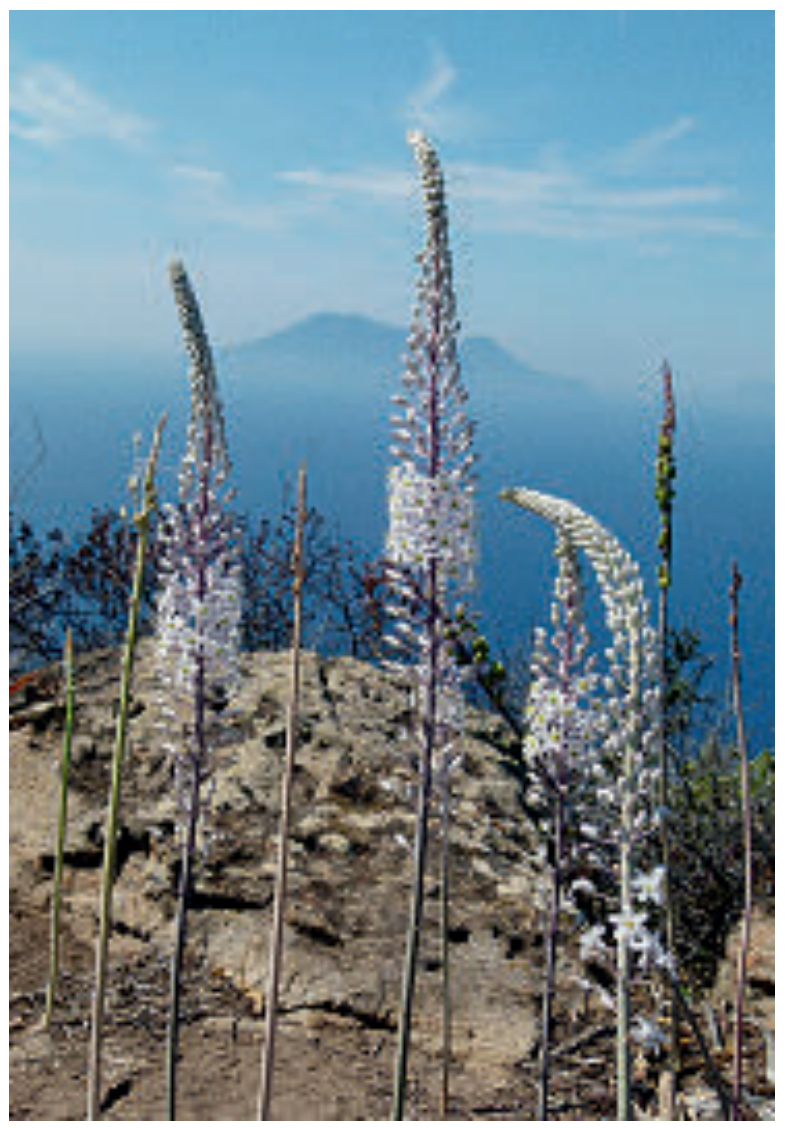

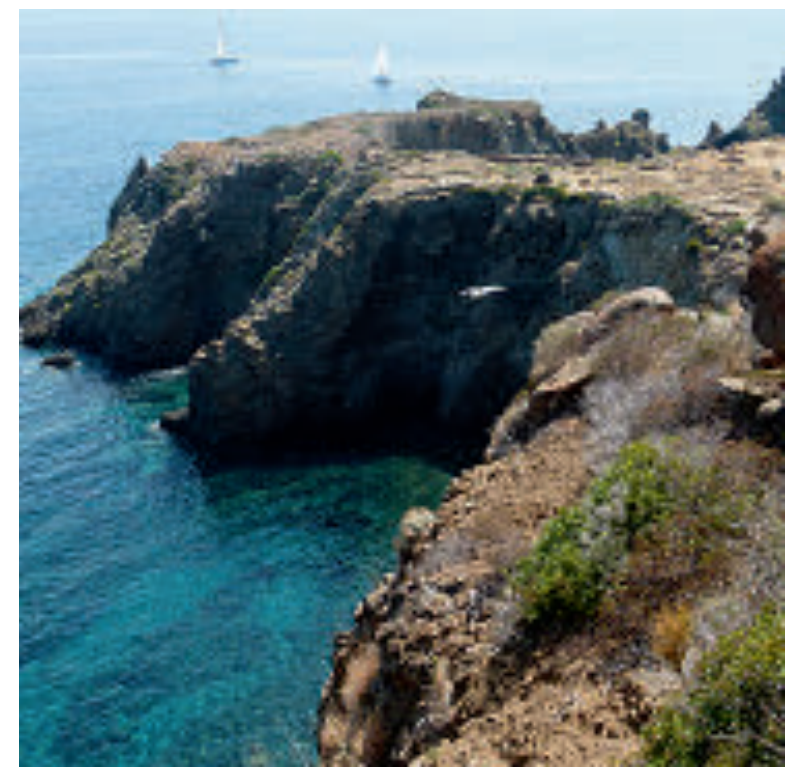

haltigste aller Liparischen Inseln. Salina ist ganzjährig ziemlich grün und steht bis auf wenige Küstenstreifen komplett unter Naturschutz.

An den Hängen der Vulkanberge haben sich immergrüne Wälder und Macchien entwickelt. Hier gibt es auch Stein-Eichen (Quercus ilex), wilde Pistazien (Pistacia terebinthus), BaumHeide (Erica arborea) und Erdbeerbäume (Arbutus unedo). An vielen Stellen wurde aber auch seit den 1960er-Jahren Akazien und Eukalypten gepflanzt, bei hohen Temperaturen ist der Duft ihrer ätherischen Öle schon von Weitem wahrzunehmen. Der Wald reicht bis zum Gipfel des Monte Fossa. Eine besonders schöne Wanderung führt von Rinella nach Pollara an der Küste entlang der Flanke des Monte dei Porri, wobei alte Bestände der Baumheide passiert werden. Der z. T. üppige Flechtenbewuchs an den Gehölzen lässt erkennen, dass es hier häufiger regnet oder zumindest Nebel für hohe Luftfeuchtigkeit sorgt. Auf Lichtungen oder Brandstellen kommt nicht selten die Meerzwiebel (Urginea martitima) vor, die im September überall ihre langen traubigen Blütenstände mit vielen weißen Blüten in die Höhe streckt. Die Blütezeit der einzelnen Individuen ist zeitlich deutlich versetzt, gleichzeitig kann man im September Exemplare finden, die gerade erst austreiben, richtig blühen oder schon Früchte angesetzt haben (Schönfelder \& SchöNFELDER 2008). 
Im fruchtbaren Tal zwischen den beiden großen Vulkanen liegen das Städtchen Malfa und die Wallfahrtskirche Madonna del Terzito. Hier - wie auch auf Lipari - wird die MalvasiaRebe angebaut. Sie ergibt einen hochwertigen, süßen Weißwein, der weit über die Inseln hinaus bekannt ist. Eine weitere wichtige Nutzpflanze auf Salina ist der Kapernstrauch (Capparis spinosa). Kapern werden hier entweder angebaut oder in der Natur von wild wachsenden Pflanzen gesammelt. Von Salina stammen die hochwertigsten Kapern überhaupt. Zur Blütezeit wird jährlich am 1. Juni-Wochenende in Malfa ein Kapernfest gefeiert (Steinecke \& Schubert 2015).

Auch Tierfreunde kommen auf Salina auf ihre Kosten. Denn hier gibt es noch einen kleinen Bestand des Eleonoren-Falken (Falco eleonorae), der im Italienischen würdevoll Falko della Regina genannt wird. Häufig sind beeindruckend große Radnetzspinnen (Argiope loba$t a)$ in ihren Netzen anzutreffen. Die Weibchen sind deutlich größer als die Männchen und erreichen Körperlängen von $2,5 \mathrm{~cm}$. Ihre Beine sind kontrastreich hell-dunkel geringelt.

\section{Filicudi und Alicudi}

Diese beiden kleinen Inseln liegen etwas abseits westlich von Salina und werden häufig, vielleicht auch wegen ihrer ähnlichen Namen, in einem Atemzug genannt. Sie sind etwas weniger touristisch erschlossen und zeigen ursprüngliche ländliche Strukturen. Der Name Filicudi geht auf die griechische Bezeichnung Phenicusa zurück, die sich auf den ehemaligen Farnreichtum der Insel bezieht. Filicudi wurde wie Salina schon in der Bronzezeit besiedelt. Vor einigen Jahren war es noch möglich, die Insel auf einem Wanderweg zu umrunden. Heute ist dies nicht mehr möglich, da der Küstenweg stellenweise aufgrund von starken Erdrutschen zerstört wurde. Stattdessen wandert man auf den 774 m hohen Vulkanberg Monte Fossa delle Felci. Von seinem Gipfel hat man einen sehr guten Blick auf die berühmte, gut $70 \mathrm{~m}$ hohe und im Meer frei stehende Felsnadel La Canna, die von Extremkletterern aus aller Welt bezwungen wird.
Im nicht ganz so steilen Gelände sind noch alte, gepflasterte Maultierpfade erhalten, die von engagierten Bewohnern instand gehalten werden. Besonders entlang dieser Wege wachsen überall reichlich Feigenkakteen (Opuntia ficus-indica), die bei einem Besuch im Juni gelbe Blüten haben und im September reichlich rote Früchte tragen. Da die vielen Samen in den Früchten relativ groß sind, werden die Früchte von den Heimischen nur bedingt genutzt, vor allem nach Entfernen der Samen zur Herstellung von Marmelade. Auch Johannisbrotbäume (Ceratonia siliqua) sind hier als traditionelle Nutzpllanzen anzutreffen.

Neben den einheimischen Pflanzen haben sich auf Filicudi auch einige Neophyten angesiedelt wie z. B. der strauchige Blaugrüne Tabak (Nicotiana glauca) aus Südamerika oder der in China heimische Götterbaum (Ailanthus altissi$m a)$. Letztgenannter ist generell im Mittelmeerraum ein invasiver Neophyt, der sich mit Hilfe seiner vielen flugfähigen Früchte effektiv ausbreitet. Die Jungpflanzen können pro Jahr $\mathrm{Zu}$ wächse von zwei oder mehr Metern haben. Oberhalb des Ortes Filicudi gibt es größere Bestände junger Götterbäume.

\section{Panarea}

Mit nur gut $3 \mathrm{~km}^{2}$ Fläche ist Panarea die kleinste der Liparischen Inseln. Ihr vorgelagert sind mehrere kleine unbewohnte Felsinseln. Auch Panarea wurde bereits in der Bronzezeit bewohnt. Auf der Punta Milazzese kann die Ausgrabung einer bronzezeitlichen Siedlung besichtigt werden (BAUSENHARDT 2010).

Auf die Insel kommen vorwiegend Tagestouristen, ansonsten gilt sie als Feriendomizil und Treffpunkt sehr wohlhabender Italiener. Die Fähren legen in San Pietro an. PKW gibt es hier nicht, dafür können sich Touristen mit E-Wagen zum Hotel oder Strand fahren lassen. Die alten, häufig weiß getünchten Häuser in den schmalen Gassen sind sehr gepflegt, die Gärten hübsch gestaltet mit diversen Zierpflanzen wie Bougainvilleen und Prunkwinden. Manch ein Gewächs macht den Eindruck, dass es hier im maritimen Klima besonders prächtig gedeiht. Auffällig sind die kräftigen Hibiskus-Sträucher 


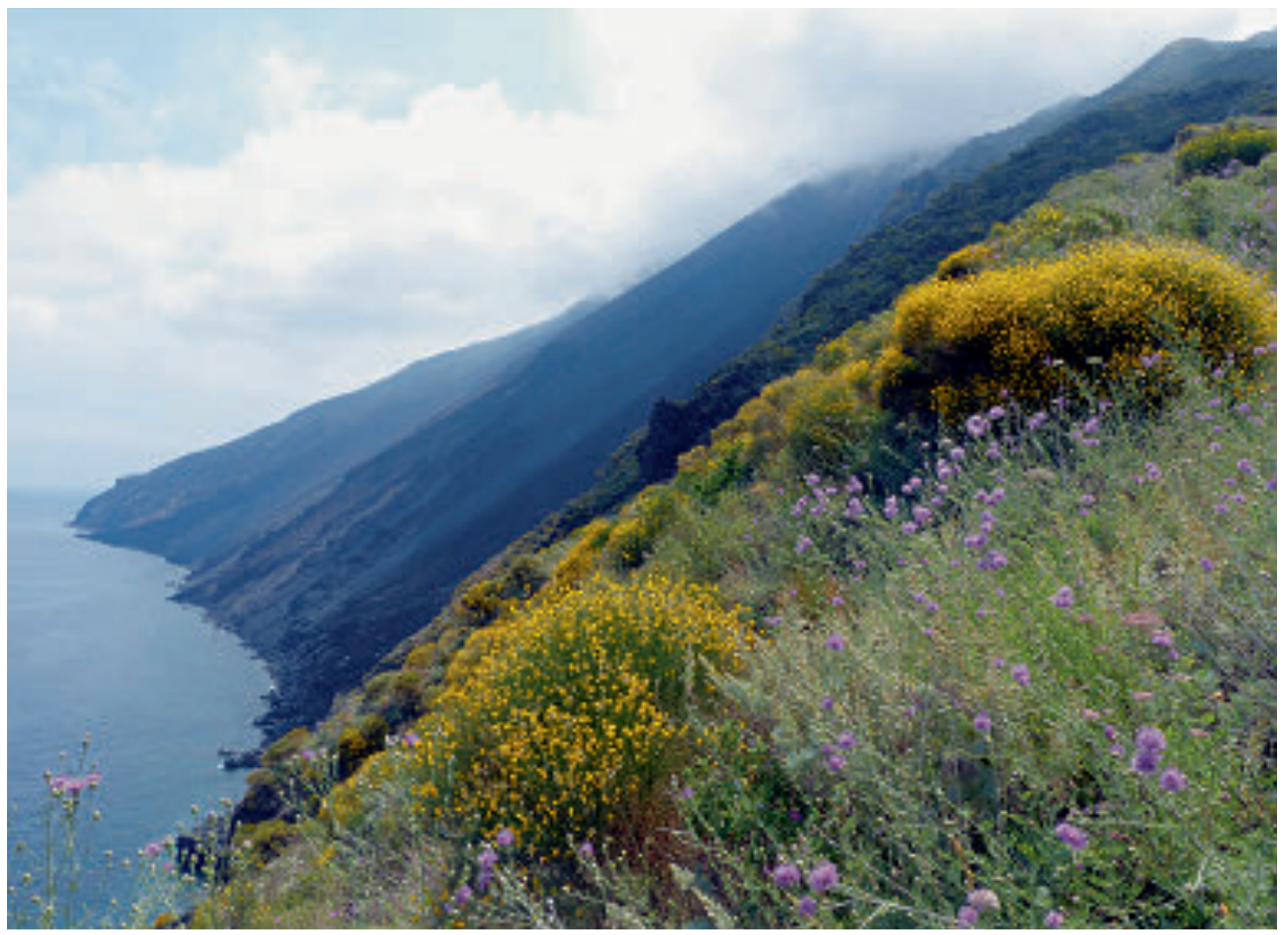

mit ihren besonders großen Blüten in Weiß, Gelb oder Rot. Weitere beliebte Zierpflanzen sind Caesalpinia pulcherrima und verschiedene Blattkakteen, die an Mauern klettern und nachts ihre großen weißen Blüten öffnen, die noch bis in die Morgenstunden geöffnet sind.

An einem einzigen Ausflugstag ist die ganze Insel leicht zu Fuß zu umrunden. Dabei führt der Weg auch zum Strand der Fumarolen, an dem an einzelnen Stellen heiße Schwefeldämpfe austreten. Von hier aus hat man einen fast unwirklichen Blick direkt auf den Stromboli, der sich wie ein Kegel mit Dampfsäule aus dem Meer erhebt. Die Wanderung führt über die Punta del Corvo (421 m), die höchste Erhebung der Insel. Zwischen den oft bizarr geformten Lavabrocken sind heimische Wildpflanzen anzutreffen. Im Herbst fällt die strauchige FelsenNelke (Dianthus rupicola) mit ihren kräftig rosa

Abb. 17: Bunt blühende Macchie auf Stromboli in der Nähe der Feuerrutsche. gefärbten, duftenden Blüten auf. Ihre Blütezeit reicht weit in den September hinein. Das Macchien-Geißblatt (Lonicera implexa) ist ein windender Halbstrauch. Es ist im Mittelmeergebiet weit verbreitet und trägt im Herbst leuchtend rote Früchte. Auf sonnigen, trockenen, auch sandigen Standorten können sich größere Bestände der Ebensträußigen Eberwurz (Carlina corymbosa) ausbreiten. Die gelben Blütenköpfe werden von diversen Insekten besucht, so auch von Holzbienen.

\section{Stromboli}

Der Höhepunkt einer Reise auf die Liparischen Inseln ist wohl der Besuch des noch aktiven Vulkans Stromboli auf der gleichnamigen Insel Stromboli. Bei einer relativ kleinen Fläche von etwa $12 \mathrm{~km}^{2}$ liegt der Gipfelbereich auf einer Höhe um 900 m, sodass die ganze Insel überall sehr steil ansteigt. Vom Meeresgrund aus betrachtet ist Stromboli sogar $3000 \mathrm{~m}$ hoch. Es handelt sich um einen klassischen Schicht- bzw. Stratovulkan. Seine Ursprünge gehen auf eine 

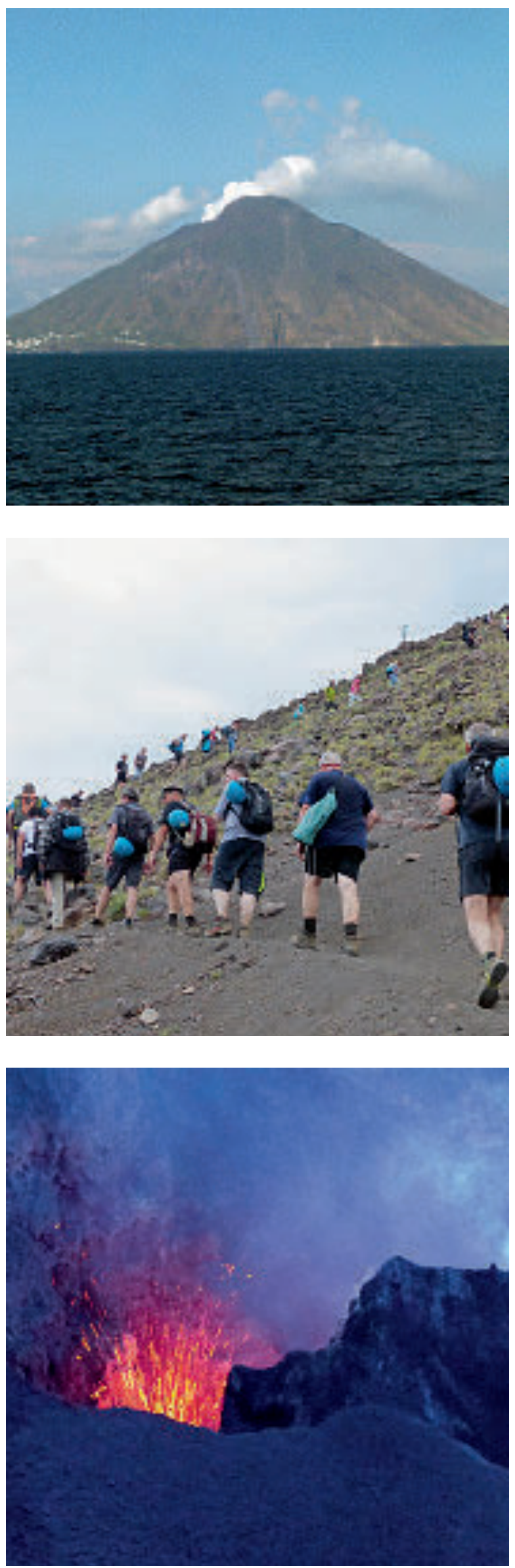

Zeit vor etwa 40000 Jahren zurück. Auf dem „Ur-Stromboli“ entstanden in zwei Phasen neue Vulkane. Der heutige Stromboli bildete sich vor etwa 5000-6000 Jahren. Das Besondere ist seine kontinuierliche Aktivität. Aus mehreren kleinen Krateröffnungen innerhalb des Zentralkraters werden in unregelmäßigen Abständen (im Minuten- bis Stundentakt) Lava, Schlacke und Gestein ausgeworfen, was sich mit einem lauten Grollen aus der Tiefe ankündigt. Die Lava fällt meist wieder in den Krater zurück oder fließt über die Feuerrutsche (Sciara del Fuoco) bis ins Meer. Die Eruptionen können durchaus $100 \mathrm{~m}$ Höhe erreichen, sodass sie vom Meer vom Schiff aus beobachtet werden können. Die kontinuierliche Aktivität des Strombolis ist weltweit einzigartig und wird als Strombolische Aktivität bezeichnet. Der Stromboli ist der aktivste Vulkan Europas.

Früher konnte man auf eigene Faust bis zum Kraterrand wandern, dort das natürliche Feuerwerk beobachten und möglicherweise auch übernachten. Aus Naturschutz- und Sicherheitsgründen ist das heute nicht mehr möglich. Ohne Bergführer ist es nur erlaubt, bis auf eine Höhe von $400 \mathrm{~m}$ aufzusteigen, ansonsten drohen empfindliche Geldstrafen. Meist vom Ort Stromboli aus gibt es aber organisierte Touren mit Bergführern. Zeitlich versetzt starten am frühen Abend mehrere Gruppen, sodass der Kraterrand bei Dunkelheit erreicht wird. Im äußerst zügigen Tempo führen die Bergführer eine regelrechte Menschenkette zum Kraterrand. Es werden nur so viele Personen zugelassen, wie sich im Ernstfall bei starkem Lavaausstoß auch in Unterständen schützen könnten. Seit Errichtung der Schutzeinrichtungen war dies bisher glücklicherweise noch nie nötig. Im Sommer 2014 war der Stromboli kurzeitig relativ stark aktiv, über die Feuerrutsche zogen sich vier Lavaströme ins Meer. Seitdem zeigte der

Abb. 18 (oben): Stromboli.

Abb. 19 (Mitte): Massenwanderung zum Kraterrand des Stromboli.

Abb. 20 (unten): Relativ schwache Eruption im Stromboli-Krater. 
Vulkan über längere Zeit eher eine schwache Aktivität mit nur sehr niedrigen Eruptionen und so gut wie keiner Lava auf der Feuerrutsche. Aber allein die Dampfsäule, die permanent aus dem zentralen Krater aufsteigt und auch aus großer Entfernung sichtbar ist, macht Stromboli zu einem faszinierenden „Bilderbuchvulkan".

Der Ort Stromboli ist von Touristen aus aller Welt überlaufen. Viel ursprünglicher ist es jedoch, den kleinen Ort Ginostra im Südwesten der Insel aufzusuchen und dort auch zu übernachten. Zwischen Stromboli und Ginostra gibt es keine Landverbindung, beide Orte sind nur über Fähren miteinander verbunden.

In Ginostra taucht der Besucher in eine Welt wie aus vergangenen Zeiten. In dem kleinen, sehr beschaulichen Ort gibt es weder Autos noch E-Karren, zudem nur zwei kleine Läden für die notwendigsten Lebensmittel. Da sich die Häuser und privaten Ferienunterkünfte steil bis auf den Timpone hinaufziehen, werden Besucher am Fähranleger gegebenenfalls mit einem Esel abgeholt, der das Gepäck den steilen, steinigen Weg hinaufträgt. Rund um Ginostra kann man entweder einen einsamen beeindruckenden Lavastrand mit bizarren Felsformationen und kleiner Lavahöhle erkunden oder auf einem schmalen Pfad bis zum Blick auf die Feuerrutsche wandern.

Stromboli ist relativ trocken und hat keine Süßwasserquellen. Regenwasser wird in Zisternen gesammelt. Im Bereich bis $100 \mathrm{~m}$ Höhe liegen die Siedlungen und wird etwas Landwirtschaft betrieben, es gibt hier u.a. auch Weinreben und Ölbäume. In den Privatgärten wachsen Feigenbäume, Zitronen und Granatapfelbäume, unter den Zierpflanzen findet man Aloen, Aeonien oder Pelargonien. Früher wurde auf Stromboli Ackerbau bis in Höhenlagen um $600 \mathrm{~m}$ betrieben, was alte aufgegebene Terrassen noch erahnen lassen. Hier breitet sich heute eine Macchien-Vegetation aus, in der häufig auch das Spanische Rohr (Arundo donax), ein großes schilfartiges Gras, anzutreffen ist. Gelegentlich wird diese Zone hier auch als Schilfzone bezeichnet. Im Frühsommer zeigt sich die Macchien-Vegetation von ihrer schönsten Seite.
Verschiedene Ginster (z.B. Spartium junceum, Genista tyrrhena) kontrastieren mit ihren gelben Blüten gegen das blaue Meer. Dazwischen gibt es viele Zistrosen sowie Wilde Möhren und die silbriggrauen Blätter des Strauch-Wermuts schimmern (Artemisia arborescens). Immer wieder entdeckt man auch die rosa Blütenköpfchen von Centaurea aeolica, einer auf den Liparischen Inseln endemischen Flockenblume.

Oberhalb von $600 \mathrm{~m}$ ist Stromboli fast vegetationsfrei aufgrund der häufigen Eruptionen mit starken Ascheauswürfen und Bildung giftiger Gase. Gerade beim Abstieg vom Stromboli wird das deutlich, wenn man durch dicke staubige Ascheschichten den Berg herunterrutscht.

Stromboli hat seit langer Zeit Menschen fasziniert. Jules Verne hat in seinem Roman „Die Reise zum Mittelpunkt der Erde“ aus dem Jahr 1864 den Stromboli aufgegriffen. Denn nach ihrer Reise ins Innerste der Erde gelangen die Expeditionsmitglieder durch den Krater des Strombolis wieder zurück an die Erdoberfläche.

Die Liparischen Inseln sind vom Frühling bis in den Herbst unbedingt eine Reise wert. Vor allem an Vulkanismus Interessierte und Naturbegeisterte kommen hier voll auf ihre Kosten. Große Hotels für den Massentourismus gibt es hier nicht und wer Ruhe und Beschaulichkeit sucht, findet sie auf den Liparischen Inseln garantiert. Durch die vor allem im Sommerhalbjahr häufigen Fährverbindungen wird das Inselhopping leicht gemacht.

\section{Literatur}

Bausenhardt, H. 2010: Marco Polo Reiseführer Sizilien, Liparische Inseln. - Ostfildern.

Schönfelder, I \& Schönfelder, P. 2008: Die neue Kosmos-Mittelmeerflora. - Stuttgart.

Steinecke, H. \& Schubert, P. 2015: Gewöhnlicher Kapernstrauch, eine Gewürzpflanze der mediterranen Küche. - Palmengarten 79: 39-41.

\section{Internetseiten}

http://www.infoeolie.com/aolischen-inseln/die-vegetationder-aolischen-inseln/

https://de.wikipedia.org/wiki/Liparische_Inseln

http://www.amapanarea.it/flora.asp

http://www.vulkane.net/vulkane/stromboli/stromboli.html 